\title{
HFS-Nullfeld-Elektronenspinresonanz an CH-Radikalen
}

\author{
M. Birkle und G. Schoffa \\ Physikalisches Institut der Universität (T. H.) Karlsruhe \\ (Z. Naturforsch. 23 a, 918-926 [1968] ; eingegangen am 22. März 1968)
}

\begin{abstract}
HFS-Nullfeldresonanz ist Elektronenspinresonanz im Feld eines Kernspins ohne äußeres Magnetfeld. Sie bietet eine Möglichkeit, den Hyperfeinkopplungstensor, d. h. die Konstanten der magnetischen Kopplung zwischen Elektronenspin und Kernspin, direkt aus dem Nullfeldspektrum der polykristallinen bzw. amorphen Substanz zu bestimmen. Die Übergangsfrequenzen liegen z. B. bei Kopplungen zu Protonen im Bereich bis ca. $200 \mathrm{MHz}$. Es wird hier der Aufbau eines Nullfeldspektrometers für Frequenzen zwischen $12 \mathrm{MHz}$ und $80 \mathrm{MHz}$ beschrieben. Die Anwendungsmöglichkeiten der HFS-Nullfeldresonanz werden an Hand von Messungen an bestrahlten Substanzen diskutiert, wobei in den vorliegenden Fällen die Aufklärung der Strahlenschäden im Vordergrund steht.
\end{abstract}

Die Hyperfeinstruktur(HFS) -Nullfeldresonanz ist ein spektroskopisches Meßverfahren zur Untersuchung der Wechselwirkung zwischen den magnetischen Momenten von ungepaarten Elektronen und den benachbarten Atomkernen. Während bei der allgemein mehr bekannten Elektronenspinresonanz (ESR) die Spin-entarteten Zustände von ungepaarten Elektronen vom äußeren Magnetfeld aufgespalten werden und die HFS-Aufspaltung nur ein Effekt zweiter Ordnung ist ${ }^{1}$, beobachtet man bei der HFS-Nullfeldresonanz direkt die HFS-Aufspaltung der Spinzustände durch die Magnetfelder der Atomkerne.

In der Ausdrucksweise der Quantenmechanik mißt die HFS-Nullfeldresonanz die Übergänge zwischen den Eigenwerten des Hamilton-Operators

$$
\mathcal{H}=\sum_{j} \mathbf{S} T_{j} \mathbf{I}_{j}
$$

mit $\mathbf{S}$ und $\boldsymbol{I}_{j}$ als Elektronenspin- bzw. Kernspinoperatoren und $T_{j}$ als HFS-Kopplungstensor des ungepaarten Elektrons zum Kern $j$.

Die ersten Vorschläge zur HFS-Nullfeldresonanz wurden 1959 von McConnell, Thompson und FesSENDEN $^{2}$ gemacht. 1963 gelang es dann Cole, KuSHIDA und Heller ${ }^{3}$, von einigen Substanzen vollständige HFS-Nullfeldspektren zu erhalten. Eine zweite Veröffentlichung über HFS-Nullfeldresonanz bei bestrahlten Glycin-Peptiden erschien $1965^{4}$.

Der Frequenzbereich, in dem die HFS-Nullfeldresonanz zu erwarten ist, hängt von der Größe der Komponenten $T_{x}, T_{y}, T_{z}$ der Kopplungstensoren ab.

1 G. Schoffa, Elektronenspinresonanz in der Biologie, BraunVerlag, Karlsruhe 1964, mit Literaturnachtrag bis 1966.

2 H. M. McConnell, D. D. Thompson u. R. W. Fessenden, Proc. Nat. Sci. U.S. 45, 1600 [1959].
Bei den meisten organischen Radikalen liegen diese zwischen 1 und $200 \mathrm{MHz}$. Die durch die ionisierenden Strahlen meist erzeugten Radikale vom Typ $\mathrm{R}_{1}-\mathrm{CH}-\mathrm{R}_{2}$ haben oft HFS-Kopplungskonstanten im Bereich von ca. $10 \mathrm{MHz}$ bis $80 \mathrm{MHz}$.

Im Verlauf dieser Arbeit wurde ein HFS-Nullfeldresonanz-Spektrometer im Frequenzbereich von $12-80 \mathrm{MHz}$ aufgebaut und die HFS-Kopplungstensoren mehrerer organischer Radikale untersucht. Ein Teil der hier ausführlich behandelten Ergebnisse ist von uns in kurzer Form bereits mitgeteilt worden *.

\section{Apparatur}

Ein Nullfeldspektrometer besteht aus einem frequenzvariablen Hochfrequenzgenerator, einem Hochfrequenzdetektor mit frequenzunabhängiger Empfindlichkeit, einem Niederfrequenzverstärker mit Lock-inGleichrichter und einem Modulationssystem. Als Hochfrequenzteil wurde ein frequenzvariabler Autodyndetektor verwendet, ähnlich wie ihn CoLE, Kushida und HELler ${ }^{3}$ vorgeschlagen haben. Durch eine Regelschaltung läßt sich eine konstante Amplitude über den ganzen Frequenzbereich erreichen. Der frequenzbestimmende Teil des Oszillators ist ein Schwingkreis, bestehend aus einem Drehkondensator und der die Probe enthaltenden Spule. Um den Bereich von $12 \mathrm{MHz}$ bis $80 \mathrm{MHz} \mathrm{zu}$ erfassen, sind verschiedene Probespulen notwendig. Da sich die einzelnen Probespulen in der Windungszahl und damit auch in der Güte unterscheiden, wird ein Intensitätsvergleich nur dadurch möglich, daß sich die Frequenzbereiche der einzelnen Spulen weit überlappen und man gleiche Linien mit verschiedenen Probespulen aufzeichnen kann. Die relativen Inten-

3 T. Cole, T. Kushida u. H. C. Heller, J. Chem. Phys. 38, 2915 [1963].

4 R. S. Mangiaracina, Rad. Res. 26, 343 [1965].

* Erscheint demnächst in J. Chem. Phys. 
sitäten der einzelnen Linien bei den gemessenen Spektren sind auf diese Weise festgelegt worden und daher nur in grober Näherung richtig. Die Tatsache, daß für den zu erfassenden Bereich von $12 \mathrm{MHz}$ bis $80 \mathrm{MHz}$ verschiedene Probespulen nötig sind und die Nullfeldspektren sich daher aus verschiedenen Teilspektren zusammensetzen, erklärt die Nichtlinearität in der Frequenzeinteilung der gezeigten Spektren. Die Frequenz selbst wird während des Meßvorganges durch Vergleich mit einem quarzstabilisierten Generator der Firma Rhode und Schwarz, Type WIK, bestimmt.

Der Oszillator ist an einen HF-Verstärker angekoppelt, an dessen Ausgang die HF-Spannung über eine Diode gleichgerichtet wird. Moduliert wird mit einer sogenannten asymmetrischen Rechteckmodulation ${ }^{5}$. Damit ergibt sich nach der Gleichrichtung über eine Lockin-Schaltung das wahre Absorptionssignal. Abb. 1 zeigt das Blockschaltbild des verwendeten Nullfeldspektrometers.

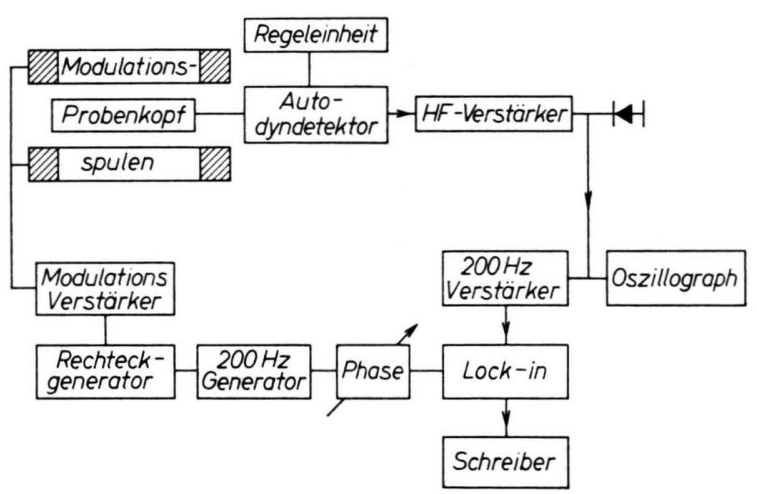

Abb. 1. Blockschaltbild des HFS-Nullfeldspektrometers.

Die Nullfeldresonanz hat gegenüber den Experimenten bei hohem äußerem Feld einige Vorteile, aber auch verschiedene Nachteile ${ }^{3}$. Als Vorteil bietet sich:

1. Das Nullfeldspektrometer benötigt keinen Magnet und kann deshalb mit weniger materiellem Aufwand gebaut werden.

2. Die Frequenzen der HFS-Nullfeldübergänge liegen im Bereich von $1 \mathrm{MHz}$ bis einigen hundert $\mathrm{MHz}$, es können deshalb auch solche Substanzen in gröBeren Volumina untersucht werden, die im Mikrowellenbereich hohe dielektrische Verluste aufweisen.

3. Da kein äußeres Magnetfeld vorliegt, treten in dem beschreibenden Hamilton-Operator keine Glieder auf, die eine bevorzugte Quantisierungsrichtung enthalten. Die Energieniveaus sind also richtungs-

5 F. Reif u. E. M. Purcell, Phys. Rev. 91, 631 [1953].

6 A. Lösche, Kerninduktion, VEB Deutscher Verlag der Wissenschaften, Berlin 1957. unabhängig gegenüber einem durch das Labor gegebenen Koordinatensystem. Prinzipiell können deshalb alle drei Hauptwerte des Hyperfeinkopplungstensors aus einem einzigen Spektrum der polykristallinen Substanz ermittelt werden.

4. Die Breite einer einzelnen Nullfeldlinie ist wesentlich geringer als im Hochfeld. Die Einflüsse, die einen Beitrag zur Linienbreite im Nullfeld geben, werden noch im Verlauf der Arbeit diskutiert.

Diesen Vorteilen gegenüber müssen aber auch folgende Nachteile in Kauf genommen werden:

1. Die größten Schwierigkeiten bereitet die Empfindlichkeit. Für die Leistung, die eine paramagnetische Probe im Resonanzfall absorbiert, gilt die Beziehung 6, 7

$$
P \sim \omega^{2}
$$

mit $\omega$ als Kreisfrequenz des HF-Feldes. Daraus geht hervor, daß beim Übergang von der Hochfeld-ESR mit einer Nachweisfrequenz von $10 \mathrm{GHz}$ zur Nullfeldresonanz bei ungefähr $50 \mathrm{MHz}$ ein erheblicher Empfindlichkeitsverlust, schon allein vom physikalischen Effekt her gesehen, auftritt. Daneben gehen in die Grenzempfindlichkeit noch die Güte des Resonanzbauteils, die Probengröße und das Modulationsverfahren ein. Aus einem Vergleich der Meßergebnisse geht hervor, da $\beta$ man insgesamt gegenüber einem guten $\mathrm{X}$-Band-Spektrometer mit einem Empfindlichkeitsverlust um mindestens einen Faktor $10^{5}$ rechnen muß.

2. Es können nur Stoffe untersucht werden, bei denen eine Aufspaltung des Elektronenzustandes durch Einflüsse der Umgebung, hier des Kernspins, vorliegt.

3. Wie noch genauer ausgeführt wird, nimmt die Zahl der Nullfeldlinien sehr stark mit der Zahl der Kernspins zu, die mit dem ungepaarten Elektron in Wechselwirkung stehen. Bei mehreren Kernspins werden die Spektren kompliziert und sind schwer zu deuten.

Die aufgeführten Vor- und Nachteile weisen darauf hin, daß Nullfeldspektroskopie auf bestimmte Substanzen beschränkt bleibt und daher als gute Ergänzung, nicht aber als Ersatz der Hochfeldspektroskopie angesehen werden kann.

7 M. BiRKLE, Diplomarbeit, Karlsruhe 1966, unveröffentlicht. 


\section{Bestimmung von Hyperfeinkopplungstensoren}

\section{A. Theoretische Grundlagen}

Der Hyperfeinstrukturkopplungstensor $\mathbf{T}$ kann in einen isotropen und in einen anisotropen Teil

zerlegt werden.

$$
\mathbf{T}=a \mathbf{U}+\mathbf{D}
$$

$\mathbf{U}$ ist dabei der Einheitstensor und $a$ ist die isotrope Kopplungskonstante. Der isotrope Kopplungsanteil der Hyperfeinstrukturaufspaltung bei Radikalen der Form - $\dot{\mathrm{C}} \mathrm{H}$ - läßt sich nach McConnelL u. a. ${ }^{8-10}$ wie folgt erklären:

Die $\dot{\mathrm{C}} \mathrm{H}$-Gruppe enthält ein ungepaartes $\pi$-Elektron am Kohlenstoffatom, der Wasserstoff ist durch eine $\sigma$-Bindung an den Kohlenstoff gebunden. Die isotrope Hyperfeinstrukturaufspaltung beruht auf einer $\sigma-\pi$-Elektronenaustausch-Wechselwirkung. Für den isotropen Anteil des Kopplungstensors gilt nun nach McConnelu ${ }^{8-10}$

$$
a=\frac{1}{3}\left(T_{x x}+T_{y y}+T_{z z}\right)=Q \varrho_{\pi}
$$

$Q$ ist dabei eine halbempirische Konstante, deren Größe z. B. für aromatische Radikale $Q=-63 \mathrm{MHz}$ beträgt. $\varrho_{\pi}$ stellt die Spindichte des ungepaarten $\pi$ Elektrons am Kohlenstoff dar.

Der anisotrope Teil $\mathbf{D}$ ist ein Tensor, dessen Spur gleich Null ist.

Dieser Kopplungsanteil kommt von einer DipolDipol-Wechselwirkung zwischen den magnetischen Momenten von Kern und Elektron. Eine Spin-BahnWechselwirkung braucht bei dem betrachteten Radikaltyp nicht berücksichtigt werden, da man eventuelle Beiträge des resultierenden Bahndrehimpulses zu der Hyperfeinaufspaltung vernachlässigen kann.

\section{B. Wechselwirkung des Elektrons mit einem Kern mit Spin 1/2}

Wenn das ungepaarte Elektron nur mit einem Proton wechselwirkt, vereinfacht sich der anfangs erwähnte Hamilton-Operator zu

$$
\mathcal{H}=S_{x} T_{x x} I_{x}+S_{y} T_{y y} I_{y}+S_{z} T_{z z} I_{z} .
$$

In diesem einfachsten Fall kann man verhältnismäßig einfach die Hamilton-Matrix aufstellen und die Frequenzen der Übergänge bestimmen, indem man vom Basisfunktionensystem

$$
\begin{aligned}
& |\alpha(\mathrm{e}) \alpha(\mathrm{p})\rangle,|\beta(\mathrm{e}) \beta(\mathrm{p})\rangle,|\alpha(\mathrm{e}) \beta(\mathrm{p})\rangle,|\beta(\mathrm{e}) \alpha(\mathrm{p})\rangle \\
& { }^{8} \text { H. M. McConnell u. D. B. Chesnut, J. Chem. Phys. 28, } 107 \\
& \text { [1958]. }
\end{aligned}
$$

mit $\alpha \rightarrow\left(\begin{array}{l}1 \\ 0\end{array}\right) \quad \beta \rightarrow\left(\begin{array}{l}0 \\ 1\end{array}\right)$ ausgeht. Damit ergeben sich die Eigenwerte und die Eigenfunktion der HamiltonMatrix :

$E_{1}=\frac{1}{4}\left(T_{z z}+T_{x x}-T_{y y}\right), \quad|1\rangle=\sqrt{\frac{1}{2}}(|\alpha \alpha\rangle+|\beta \beta\rangle)$, $E_{2}=\frac{1}{4}\left(T_{z z}-T_{x x}+T_{y y}\right), \quad|2\rangle=\sqrt{\frac{1}{2}}(|\alpha \alpha\rangle-|\beta \beta\rangle)$, $E_{3}=\frac{1}{4}\left(-T_{z z}+T_{x x}+T_{y y}\right), \quad|3\rangle=\sqrt{\frac{1}{2}}(|\alpha \beta\rangle+|\beta \alpha\rangle)$, $E_{4}=\frac{1}{4}\left(-T_{z z}-T_{x x}-T_{y y}\right),|4\rangle=\sqrt{\frac{1}{2}}(|\alpha \beta\rangle-|\beta \alpha\rangle)$.

Zwischen diesen vier Energieniveaus ist jeder Übergang erlaubt, sofern seine Polarisationsrichtung mit der des HF-Feldes übereinstimmt. Bei einer polykristallinen Substanz kann man also 6 Absorptionslinien beobachten. Mit Hilfe der Auswahlregeln $\Delta m=0, \pm 1$ ergibt sich, daß jeweils zwei Übergänge $x$-, $y$ - bzw. $z$-polarisiert sind.

Auf der Abb. 2 sind typische Spektren für Einprotonwechselwirkung zu sehen. Aus diesen Spektren lassen sich nun direkt und sehr genau die

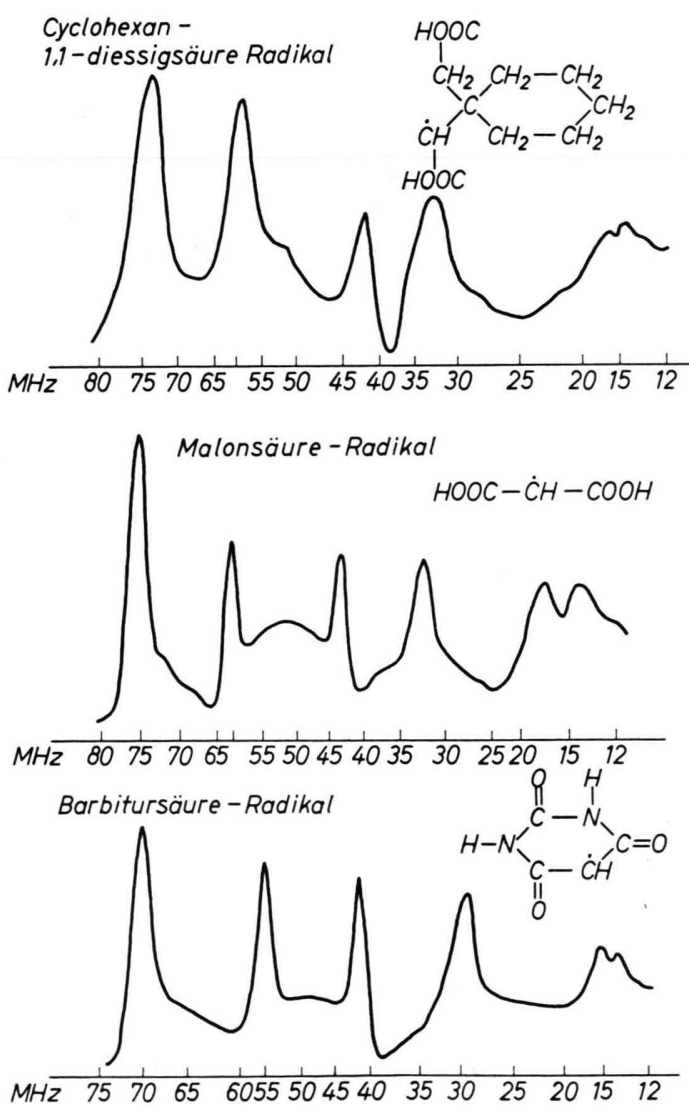

Abb. 2. Spektren für Einprotonenwechselwirkung.

9 H. M. McConnell, J. Chem. Phys. 24, 764 [1956].

10 H. M. McConnell, J. Chem. Phys. 28, 1188 [1958]. 
Hauptwerte des Kopplungstensors $T_{x x}, T_{y y}, T_{z z}$ ermitteln. Zur Berechnung der drei Tensorwerte genügen die Linienfrequenzen von 3 Linien. Die weiteren Linien können daher als Kontrolle des Meßverfahrens und der Zuordnung der Linienfrequenzen zu den jeweiligen Übergängen dienen. Die beiden Linien mit den niedersten Frequenzen liegen eng beieinander und sind deshalb nicht immer aufgelöst. Tab. 1 gibt die aus den drei höchsten Linienfrequenzen berechneten Werte für den Hyperfeinkopplungstensor und für den darin enthaltenen isotropen Anteil $a$ wieder. Zum Vergleich sind in der letzten Spalte die Hochfeldwerte angegeben, soweit sie bekannt sind ${ }^{11,12}$. Bei der Berechnung der Nullfeldwerte wurde, von den Hochfeldwerten herkommend, die Konvention $\left|T_{y y}\right|>\left|T_{x x}\right|>\left|T_{z z}\right|$ zugrunde gelegt. Weiterhin wurde angenommen, daß alle $T_{i i}$ das gleiche Vorzeichen (negativ) tragen. Die absoluten Vorzeichen der Tensorwerte können mit der HFSNullfeldresonanz allein nicht bestimmt werden. Die angegebenen Nullfeldwerte in Tab. 1 sind auf $\pm 0,5$ $\mathrm{MHz}$ genau. Die eingeklammerten Nullfelddaten für Malonsäure stammen aus der Arbeit von Cole, $\mathrm{KU}$ SHIDA und HELLER ${ }^{3}$.

Bei verschiedenen Spektren, vor allem bei den in Abb. 2 gezeigten Spektren, tritt eine Nullinienschwankung mit deutlichem Minimum bei $40 \mathrm{MHz}$ auf. Wahrscheinlich handelt es sich hierbei um ein überlagertes Signal, dessen Herkunft nicht geklärt werden konnte. Feststellen ließ sich lediglich, daß weder eine rein apparative Nullpunktschwankung noch Kernquadrupolresonanzlinien als Ursache in Frage kommen.

\section{Linienbreite, Linienform}

Die Spektren der Abb. 2 zeigen Linienbreiten in der Größenordnung von $1 \mathrm{MHz}$ bis $3 \mathrm{MHz}$, dagegen liegen die entsprechenden Linienbreiten bei Hochfeldmessungen bei ungefähr $10 \mathrm{MHz}^{12,13}$. Da die Energieniveaus in Abschnitt B nicht entartet sind, besitzt das Radikal kein magnetisches Moment in erster Näherung und in dieser Näherung tritt auch keine magnetische Wechselwirkung mit irgendwelchen lokalen Feldern im Kristall auf. Wenn man annimmt, daß die benachbarten, von Null verschiedenen Kernspins ein lokales magnetisches Feld $B_{\text {loc }}$ erzeugen, das für die Linienbreite verantwortlich ist, so ergibt erst die zweite Näherung der Störungsrechnung einen von Null verschiedenen Beitrag. Die Breite der Nullfeldlinien liegt damit in der Größenordnung von $1 \mathrm{MHz}$ bis $2 \mathrm{MHz}$. Bei sehr vielen Spektren erhält man jedoch wesentlich breitere Linien. Diese Verbreiterung geht meist darauf zurück, daß durch Wechselwirkung des ungepaarten Elektrons mit mehreren Kernspins weitere Linien entstehen, die im einzelnen nicht aufgelöst werden. Dieser Effekt tritt nach Heller ${ }^{14}$ hauptsächlich dann auf, wenn man eine Kopplung eines Kerns mit $a_{1}$ und eines zweiten Kerns mit $a_{2}$ derart hat, daß $a_{1} / 4>a_{2}>-a_{1} / 4$ ist. Die Linien auf Grund der Aufspaltung $a_{1}$ erscheinen dann durch die zusätzliche Aufspaltung $a_{2}$ verbreitert. So geht zum Beispiel die Linienverbreiterung der Spektren in Abschnitt E auf einen solchen Effekt zurück. Auch die leichte Verbreiterung der Linien der Cyclohexan-1,1-diessigsäure geht wahrscheinlich auf eine schwache Wechselwirkung mit Protonen in $\gamma$-Stellung zurück.

Eine weitere Möglichkeit für Linienverbreiterung liegt vor, wenn die Nullfeldniveaus entartet sind und diese Entartung durch ein nicht vollständig verschwindendes Erdfeld oder dgl. teilweise aufgehoben wird und damit zusätzlich Linien entstehen. Im allgemeinen können schwache äußere Felder jedoch vernachlässigt werden. Der Fehler, den sie bei der

\begin{tabular}{|c|c|c|c|c|c|c|c|c|c|c|c|c|}
\hline \multirow[t]{2}{*}{ Substanz } & \multicolumn{4}{|c|}{ Linienfrequenzen in $\mathrm{MHz}$} & \multicolumn{3}{|c|}{ Nullfeldwerte (MHz) } & \multirow[b]{2}{*}{$a_{\mathrm{i}}$} & \multicolumn{4}{|c|}{ Hochfeldwerte (MHz) } \\
\hline & $V_{6}$ & $V_{5}$ & $V_{4}$ & $V_{3}$ & $T_{x x}$ & $T_{y y}$ & $T_{z z}$ & & $T_{x x}$ & $T_{y y}$ & $T_{z z}$ & $a_{\mathrm{i}}$ \\
\hline Barbitursäure & 70,2 & 54,7 & 40,9 & 29,2 & $-56,4$ & $-84,0$ & $-25,4$ & $-55,3$ & $-57,2$ & $-84,1$ & $\begin{array}{r}-31,4 \\
\text { (s. }\end{array}$ & $\begin{array}{l}-57,6 \\
\text { Anm. }\end{array}$ \\
\hline Malonsäure & 75,1 & 60,6 & 43,1 & 32,2 & $\begin{array}{r}-57,6 \\
(-56,8\end{array}$ & $\begin{array}{l}-92,6 \\
-92,4\end{array}$ & $\begin{array}{l}-28,6 \\
-28,4\end{array}$ & $\begin{array}{c}-59,6 \\
-59,2) \\
\left.\text { (s. Anm. }{ }^{3}\right)\end{array}$ & -53 & -86 & -28 (s. & Anm. 11) \\
\hline $\begin{array}{l}\text { Glyclohexan-1,1- } \\
\text { diessigsäure }\end{array}$ & 73,1 & 58,3 & 41,3 & 32,0 & $-56,1$ & $-90,1$ & $-26,5$ & $-57,6$ & & Anm. $\left.{ }^{11}\right)$ & & -58 \\
\hline
\end{tabular}

11 Landolt-Börnstein, Zahlenwerte und Funktionen, Neue Serie, Gruppe II, Band I, Magnetische Eigenschaften freier Radikale, Springer-Verlag, Berlin 1965.

12 W. Bernhard u. W. Snipes, J. Chem. Phys. 44, 2817 [1966].
13 H. M. McConnell, H. C. Heller, T. Cole u. R. W. FessenDEN, J. Am. Chem. Soc. 82, 666 [1960].

14 H. C. Heller, J. Chem. Phys. 42, 2611 [1965]. 
Bestimmung der Linienfrequenzen verursachen, läßt sich mit der BreIt-RABI-Formel ${ }^{15}$ abschätzen. Er liegt bei einem äußeren Feld von 0,3 Gauss in der Größenordnung von $1 \%$.

\section{Wechselwirkung des Elektrons mit mehreren Kernen mit Spin 1/2}

Wie schon erwähnt, wächst die Zahl der Linien bei der Nullfeldspektroskopie sehr rasch mit der Zahl der wechselwirkenden Kerne. Für den Fall von $n$ nichtäquivalenten Protonen erhält man im allgemeinen $2^{n+1}$ Energieniveaus und $2^{2 n+1}-2^{n}$ mögliche Übergänge, also bei drei Protonen bereits 120 mögliche Ưbergänge ${ }^{3}$.

Der den allgemeinen Fall beschreibende HamiltonOperator hat die Form

$$
\mathcal{H}=\sum_{j=1}^{n} S T_{j} I_{j}=\sum_{j} \sum_{i} \sum_{k} T_{j i k} S_{i} I_{j k}
$$

mit $j+=1,2, \ldots, n$ und $k$ und $i=x, y, z . T_{j i k}$ sind die Elemente des allgemeinen Hyperfein-Kopplungstensors zwischen dem Elektron und dem $j$-ten Kern. $S_{i}$ und $I_{j k}$ sind die Pauli-Matrizen.

Betrachtet man $n$ Kerne mit Spin $1 / 2$, so erhält man $g=2^{n+1}$ Basisfunktionen.

Wenn die Hyperfein-Kopplungstensoren und die Lage ihrer Diagonalsysteme zueinander z.B. aus Hochfeldexperimenten bekannt sind, kann man das zu erwartende Nullfeldspektrum mit seinen relativen Intensitäten mit Hilfe eines Computerprogramms von LefEbvre und Maruani maschinell berechnen ${ }^{16}$.

Bei berechneten Spektren wird deutlich, daß ganz erhebliche Unterschiede in den Intensitäten der einzelnen Übergänge auftreten. Es zeigt sich dabei, $\mathrm{da} ß$ die Zahl der hervorragenden Linien gering ist im Verhältnis zu den vielen möglichen Übergängen. Damit erklärt sich, warum die beobachteten Spektren sogar in Bereichen großer Liniendichte als ziemlich gut aufgelöst erscheinen.

Die aus Hochfeldexperimenten bestimmten Tensorwerte, von denen man bei der Rechnung meist ausgeht, sind vielfach mit Unsicherheiten von einigen $\mathrm{MHz}$ behaftet. Um auch in Fällen mit komplizierteren Nullfeldspektren genauere Werte für die HFS-Kopplungstensoren zu erhalten, kann man die berechneten Spektren möglichst gut an die beobachteten anpassen, indem man bei der Rechnung die Ausgangsverte geeignet variiert. Diesem Verfahren

15 G. Breit u. I. I. Rabi, Phys. Rev. 38, 2082 [1931].

sind jedoch dadurch Grenzen gesetzt, daß man im allgemeinen Fall eine große Zahl freier Parameter hat, da die Tensorwerte und die Winkel der Diagonalsysteme zueinader variiert werden müssen.

Beim einfachsten Fall der Wechselwirkung mit mehreren Protonen, d. h. bei der Wechselwirkung des Elektrons mit zwei Protonen, ergeben sich $6 \mathrm{Li}$ nien auf Grund von 6 Übergängen zwischen 4 doppelt entarteten Energieniveaus. Wenn die beiden Tensoren nicht im gleichen System diagonal sind, hat man mehr als 6 zu variierende Werte. Das Problem ist also unterbestimmt durch die Angabe von nur 6 Linienfrequenzen, da die gemessenen Intensitäten aus den am Anfang angeführten Gründen mit Fehlern behaftet sind, die in der Größenordnung der Unsicherheiten der Họchfeldwerte liegen.

Ungeachtet all dieser Schwierigkeiten bei Wechselwirkung mehrerer Kerne bietet das gemessene Nullfeldspektrum durch Vergleich mit dem aus Hochfeldwerten errechneten Spektrum immer eine gute Kontrolle der zum Teil umstrittenen Hochfeldwerte.

\section{Beispiele:}

Glykolsäure: Das durch Bestrahlung mit $2 \mathrm{MeV}$ Elektronen und $30 \mathrm{Mrad}$ von polykristalliner Glykolsäure entstehende Radikal $\mathrm{HO}-\dot{\mathrm{C}} \mathrm{H}-\mathrm{COOH}$ ist ein typisches Beispiel für die Wechselwirkung des Elektrons mit zwei nicht äquivalenten. Protonen. Die beiden Kopplungstensoren sind in verschiedenen Systemen diagonal. Abb. 3 zeigt das gemessene und das aus

Gemessenes Nullfeld-Spektrum des Radikals $\mathrm{HO}-\dot{\mathrm{CH}}-\mathrm{COOH}$

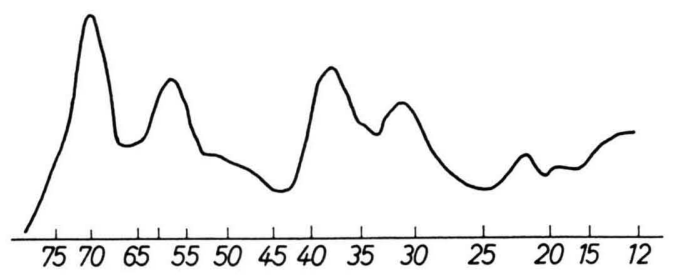

Aus Hochfeldwerten berechnetes Spektrum

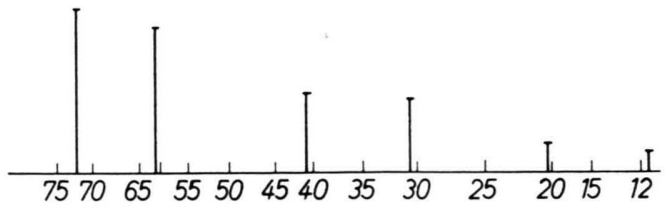

Abb. 3. Nullfeldspektren bestrahlter Glykolsäure.

16 R. Lefebvre u. J. Maruani, J. Chem. Phys. 42, 1496 [1965]. 
Hochfeldwerten ${ }^{17}$ berechnete Spektrum. Die Ubereinstimmung mit dem gemessenen Nullfeldspektrum ist nicht besonders gut. Eine Variation der Hochfeldwerte und damit eine Anpassung des berechneten an das gemessene Spektrum wurde aus den vorher genannten Gründen nicht durchgeführt.

Fumarsäure: Die in polykristalliner Form vorliegende Fumarsäure wurde mit $\gamma$-Quanten aus einer $\mathrm{Co}^{60}$. Quelle mit einer Dosis von ca. 60 Mrad bestrahlt. Dabei sollen nach CooK, Rowlands und WhIFFen ${ }^{18}$ als Radikale $\mathrm{HOOC}-\mathrm{CHR}-\dot{\mathrm{C}} \mathrm{H}-\mathrm{COOH}$ und in etwas geringerer Konzentration $\mathrm{HOOC}-\mathrm{CH}_{2}-\dot{\mathrm{C}} \mathrm{H}-\mathrm{COOH}$ entstehen. Man hat also ein Zweiprotonen- und Dreiprotonenradikal vorliegen, wobei das letztere auch bei Bestrahlung von Succinsäure entsteht. Abb. 4 zeigt das gemessene Nullfeldspektrum und die für die beiden Radikale berechneten Spektren. Der Berechnung der Linien des Hauptradikals wurden die Werte von Соок u. a. ${ }^{18}$ zugrunde gelegt. Damit ergeben sich die Frequenzen der 6 Linien zu $102,90 \mathrm{MHz}, 60,47 \mathrm{MHz}$, $60,45 \mathrm{MHz}, 42,45 \mathrm{MHz}, 42,43 \mathrm{MHz}, 18,02 \mathrm{MHz}$. Das nach Hochfeldwerten zu erwartende Spektrum des zweiten Radikals ist eines der von Lefebvre und Maruani ${ }^{16}$ gerechneten Beispiele zu ihrem Programm. Der in Abb. 4 wiedergegebene Teil dieses Spektrums enthält
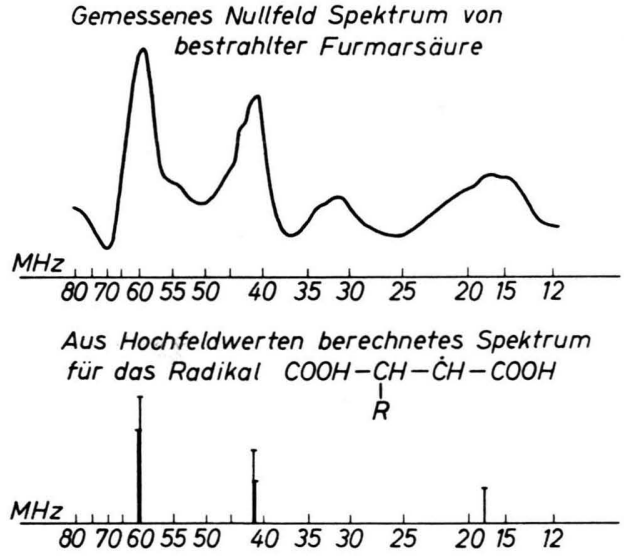

Aus Hochfeldwerten berechnetes Spektrum für das Radikal $\mathrm{COOH}-\mathrm{CH}_{2}-\mathrm{C} \mathrm{H}-\mathrm{COOH}$

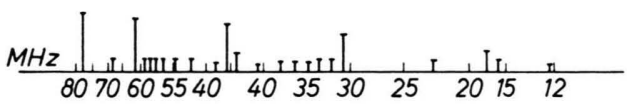

Abb. 4. Nullfeldspektren bestrahlter Fumarsäure.

davon nur die intensiveren Linien. Das Verhältnis der Intensitäten der beiden berechneten Spektren wurde an Hand des gemessenen Nullfeldspektrums festgelegt. Die Annahme, daß die gemessene Liniengruppe durch

17 N. N. Atherton u. D. H. Whiffen, Mol. Phys. 3 I [1960].

18 R. J. Сook, J. R. Rowlands u. D. H. Whiffen, J. Chem. Soc. 1963, 3520 .
Überlagerung der Spektren der beiden Radikaltypen entsteht, scheint sich durch die gute Übereinstimmung zwischen berechneten und gemessenen Werten zu bestätigen.

$\alpha$-Alanin: Bei $\gamma$-Bestrahlung von 1- $\alpha$-Alanin (ca. 30 Mrad) entsteht das Radikal $\mathrm{CH}_{3}-\dot{\mathrm{C}} \mathrm{H}-\mathrm{COOH}$. Eine Wechselwirkung des ungepaarten Elektrons mit 4 Protonen ergibt damit 32 Energieniveaus und 496 mögliche Ubergänge. Wie aus früheren Arbeiten hervorgeht ${ }^{19,20}$, kann man bei Zimmertemperatur die Kopplung der drei Methylprotonen wegen der Rotation der Methylgruppe als ungefähr gleich und isotrop annehmen. Bei der Berechnung des Nullfeldspektrums zeigt sich, wie schon erwähnt, daß von den möglichen Übergängen nur wenige mit nachweisbarer Intensität auftreten. Das gesamte Spektrum des Alaninradikals reicht von einigen $\mathrm{Hz}$ bis in die Gegend von $190 \mathrm{MHz}$. In Abb. 5 sind die im Bereich von $12 \mathrm{MHz}$ bis $80 \mathrm{MHz}$ liegenden Teile von gemessenen und berechneten Nullfeldspektren dargestellt. Voraussetzung bei der Berechnung war in beiden Fällen gleiche und isotrope Kopplung der Methylprotonen $\left(T_{i i}=70,50 \mathrm{MHz}\right)$. In einem Fall wurde jedoch für die Kopplung des Protons am Kohlenstoff die Hochfeldwerte ${ }^{19}$ und im anderen Fall geänderte Werte
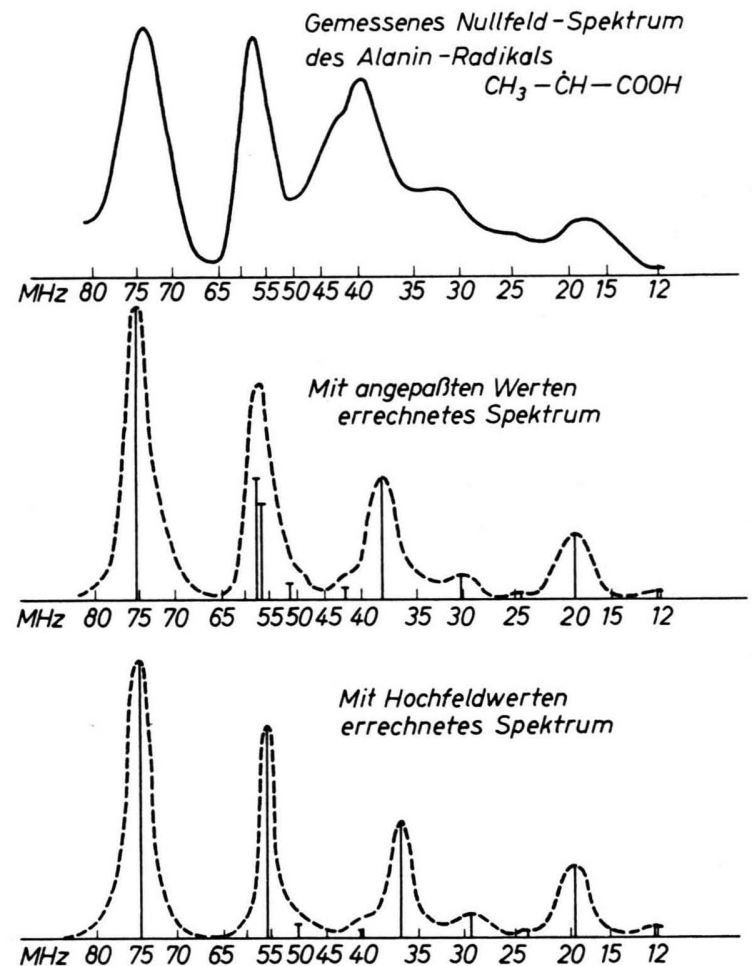

Abb. 5. Nullfeldspektren des bestrahlten $\alpha$-Alanins.

19 J. R. Morton u. A. Horsfield, J. Chem. Phys. 35, 1142 [1961].

20 I. Miyagawa u. K. Jтон, J. Chem. Phys. 36, 2157 [1962]. 
$T_{x x}=56,5 \mathrm{MHz} ; \quad T_{y y}=91,4 \mathrm{MHz} ; \quad T_{z z}=23,4 \mathrm{MHz}$ zugrunde gelegt. Das Radikal $\mathrm{CH}_{3}-\dot{\mathrm{CH}}-\mathrm{COOH}$ läßt sich auch in Lösung über eine chemische Reaktion erzeugen und in einem speziellen Durchflußverfahren untersuchen ${ }^{21,22}$. Die Werte für den isotropen Anteil $a=\frac{1}{3}\left(T_{x x}+T_{y y}+T_{z z}\right)=57,1 \mathrm{MHz}$ stimmen mit dem in Lösung bestimmten $a=56,4 \mathrm{MHz}$ innerhalb der Fehlergrenzen überein.

Bei den berechneten Spektren ist der Kurvenverlauf für eine angenommene Linienbreite von ca. $5 \mathrm{MHz}$ gestrichelt gezeichnet. Um eine noch bessere Anpassung des berechneten an das gemessene Spektrum zu erreichen, müßte man die schwache Anisotropie der Methylprotonen mit berücksichtigen. Dadurch würde aber die Zahl der möglicherweise zu variierenden Größen so stark anwachsen, daß die Berechnung der Spektren, selbst mit einer elektronischne Rechenmaschine, zu aufwendig wäre.

\section{E. Wechselwirkung des Elektrons mit Kernen mit Spin 1 und Spin 1/2}

Wie aus Hochfeldergebnissen ${ }^{11}$ hervorgeht, muß zur Deutung vieler Spektren von Substanzen, die Stickstoff enthalten, noch eine zusätzliche, wenn auch meist schwache Wechselwirkung mit einem Stickstoffkern beachtet werden.

Im allgemeinen Fall mit $n$ Kernen mit Spin $1 / 2$ und $m$ Kernen mit Spin 1 erhält man $g=2 \cdot 2^{n} \cdot 3^{m}$ Basisfunktionen und damit gleich viele Energieniveaus.

Bedauerlicherweise konnte zur Berechnung der Spektren mit Wechselwirkung zu Stickstoffkernen nicht auf ein Computerprogramm zurückgegriffen werden. Für den Fall mit einem Proton und einem Kern mit Spin 1 wurden im Verlauf dieser Arbeit die Energieniveaus und die Frequenzen der Übergänge berechnet. Als Beispiel für diesen Fall seien die Spektren von Glycylglycin, Creatinin und Glycocyamin angegeben.

\section{Beispiele:}

Glycylglycin, Glycocyamin, Creatinin: Bei $\gamma$-Bestrahlung dieser Substanzen entstehen Radikale, die alle die Gruppe $=\mathrm{N}-\dot{\mathrm{C}} \mathrm{H}-$ enthalten. Zusätzlich zur Kopplung des ungepaarten Elektrons an das $\alpha$-Proton tritt hier also noch eine schwache Wechselwirkung mit dem Stickstoffkern hinzu. Man erwartet in erster Näherung ein Spektrum mit 6 Linien, ähnlich den Spektren der Einprotonenradikale der Abb. 2. Die tatsächlich gemessenen Spektren bestehen auch aus 6, allerdings wesentlich verbreiterten Linien (Abb. 6, 7), aus deren

21 H. Fischer, Z. Naturforsch. 19 a, 866 [1964].

22 W. T. Dixon u. R. O. C. Norman, J. Chem. Soc. 1963, 3119.
Zentren sich der Kopplungstensor zum $\alpha$-Proton näherungsweise bestimmen läßt. Tab. 2 zeigt, daß die Ergebnisse von Nullfeld- und Hochfeldresonanz ${ }^{23,}{ }^{24}$ zum Teil voneinander abweichen.

a) gemessenes Spektrum des Glycocyamin-Radikals $\begin{aligned} \mathrm{HN} & =\mathrm{C}-\mathrm{NH}-\dot{\mathrm{C}} \mathrm{H}-\mathrm{COOH} \\ & \mathrm{N} \mathrm{H}_{2}\end{aligned}$

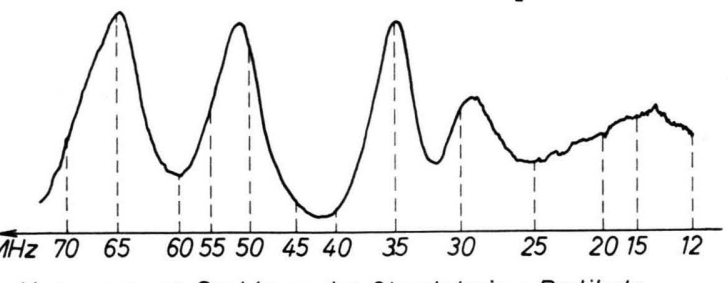

b) gemessenes Spektrum des Glycylglycin - Radikals

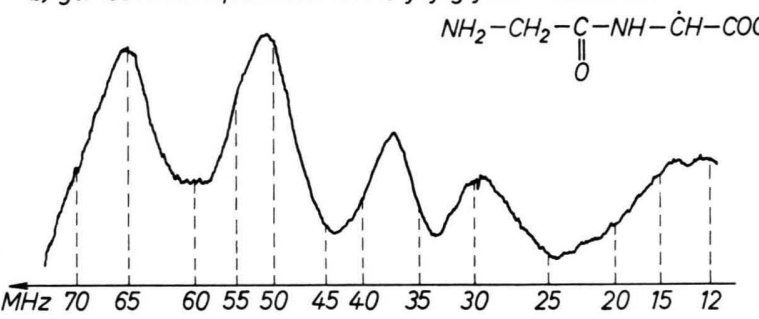

c) berechnetes Spektrum des Glycylglycin-Radikals

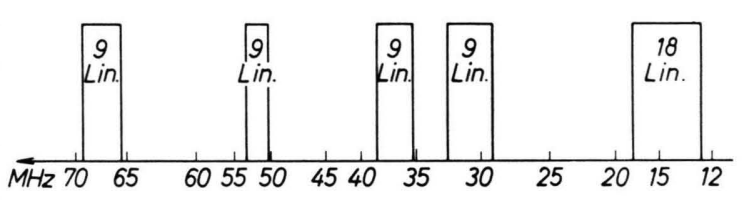

Abb. 6. Nullfeldspektren von Glycocyamin und Glycylglycin.

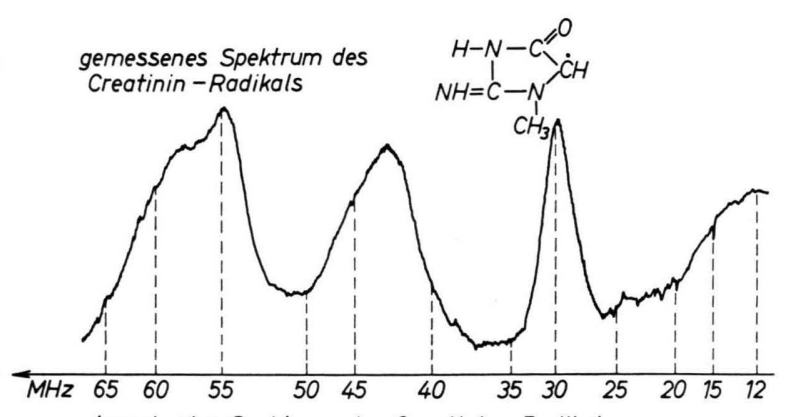

berechnetes Spektrum des Creatinin-Radikals

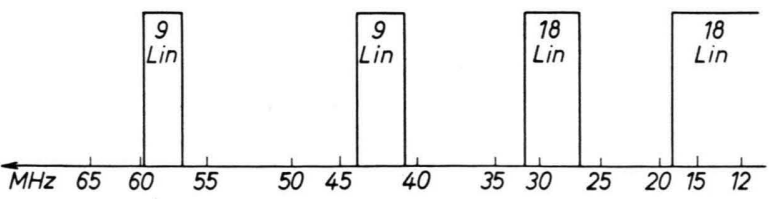

Abb. 7. Nullfeldspektren von Creatinin.

23 M. Katayama u. W. Gordy, J. Chem. Phys. 35, 117 [1961]. 24 H. Ueda, J. Chem. Phys. 40, 901 [1964]. 
Aus der Hochfeldspektroskopie sind nur für Glycylglycin die Werte für die Stickstoffkopplung bekannt ${ }^{23}$. Bei der Berechnung der Spektren unter Einbeziehung der Wechselwirkung zum Stickstoffkern zeigt sich nun, da $ß$ jede der 6 verbreiterten Linien in Wirklichkeit eine Liniengruppe darstellt, bestehend aus mehreren eng beieinanderliegenden Einzellinien. Abb. 6 und 7 zeigen neben den gemessenen auch zwei nach den Nullfelddaten der Tab. 2 berechnete Spektren.

\begin{tabular}{cllll}
\hline & & $\begin{array}{c}\text { Glycyl- } \\
\text { glycin }\end{array}$ & $\begin{array}{c}\text { Glyco- } \\
\text { cyamin }\end{array}$ & Creatinin \\
\hline & $T_{x x}(\mathrm{H})$ & $-51,7$ & $-48,0$ & $-44,3$ \\
& $T_{y y}(\mathrm{H})$ & $-79,6$ & $-80,4$ & $-69,7$ \\
Nullfeldwerte & $T_{z z}(\mathrm{H})$ & $-22,7$ & $-21,6$ & $-15,3$ \\
(in MHz) & $T_{x x}(\mathrm{~N})$ & $-11,2^{*}$ & $-11,2^{*}$ & $-11,2^{*}$ \\
& $T_{y y}(\mathrm{~N})$ & $-8,4^{*}$ & $-8,4^{*}$ & $-8,4^{*}$ \\
& $T_{z z}(\mathrm{~N})$ & $-5,6^{*}$ & $-5,6^{*}$ & $-5,6^{*}$ \\
& $T_{z z}(\mathrm{~N})$ & $-46,7^{23}$ & & $-50,5^{24}$ \\
Hochfeldwerte & $T_{x x}(\mathrm{H})$ & $-82,5$ & & $-68,0$ \\
(in MHz) & $T_{z z}(\mathrm{H})$ & $-24,1$ & & $-32,8$ \\
& $T_{x x}(\mathrm{~N})$ & $-11,2$ & & $\leqq 11,5$ \\
& $T_{y y}(\mathrm{~N})$ & $-5,6$ & & $\leqq 11,5$ \\
&
\end{tabular}

* Diese Werte lassen sich nicht direkt aus den Nullfeldspektren ermitteln. Bei allen 3 Substanzen werden die Hochfeldwerte von Glycylglycin verwendet, um die Spektren näherungsweise berechnen zu können.

Tab. 2.

Die Deutung, daß die Linienverbreiterung bei diesen Substanzen eine Wechselwirkung mit dem Stickstoffkern als Ursache hat, steht allerdings im Widerspruch zu einer früheren Nullfeldarbeit über Glycylglycin ${ }^{4}$, in der angenommen wird, daß die beiden Protonen der ursprünglichen $=\mathrm{CH}_{2}$-Gruppe nicht äquivalent sind. Bei der Bestrahlung würden demnach zwei Radikale entstehen, so daß jede der verbreiterten Linien eine Doppellinie wäre. Diese Behauptung ist mit den aufgeführten Ergebnissen zwar nicht eindeutig widerlegt, jedoch scheint die Stickstoffwechselwirkung als Ursache der Linienverbreiterung wesentlich wahrscheinlicher zu sein.

\section{Schlußbemerkung}

Die hier untersuchten Frequenzen dürften das unterste Stück auf der Frequenzskala der HFS-Nullfeldresonanz darstellen. Eine Erweiterung des Frequenzbereichs nach oben wird die Untersuchung auch von Radikalen anderer Struktur ermöglichen.

Die HFS-Elektronenspin-Nullfeldresonanz hat gegenüber der üblichen Hochfeld-ESR vor allem den Vorteil, daß man zur Bestimmung der anisotropen HFS-Kopplungen nicht auf Einkristalle angewiesen ist. Als selbständiges Meßverfahren ist sie jedoch der Hochfeldresonanz nur dann überlegen, wenn man den Fall des Einprotonenradikals vorliegen hat oder diesen in erster Näherung annehmen kann. Bei Wechselwirkung des Elektrons mit mehreren Kernen muß das zu erwartende Nullfeldspektrum, von irgendwelchen vorgegebenen Werten ausgehend, berechnet und dann durch geeignete Variation an das gemessene Spektrum angepaßt werden. Dieses Verfahren bietet theoretisch zwar genaue Werte, aber als besseres oder gleichwertiges Meßverfahren gegenüber der Hochfeldresonanz kann man in diesem Fall die Nullfeldresonanz erst dann betrachten, wenn entsprechende Computerprogramme vorliegen, so daß man die meist sehr umfangreiche Variation bzw. Anpassung maschinell durchführen kann. Die HFSNullfeldresonanz ist wegen der linienreichen Spektren mehr noch als die übliche ESR auf Computerprogramme angewiesen. Leider gibt es zum jetzigen Zeitpunkt, mit Ausnahme des Programms von LEFebvre und Maruani ${ }^{16}$ für maximal 4 Kernspins mit $I=1 / 2$, weder ein allgemeines Programm, das $I>1 / 2$ einbezieht, noch ein solches, das eine Anpassung des gerechneten an ein gemessenes Spektrum erlaubt. Ein Programm für die Berechnung der Nullfeldspektren bei einem Kernspin $I=1$ und maximal 4 Kernspins $I=1 / 2$ dürfte allerdings in absehbarer Zeit zur Verfügung stehen. Am „Centre de Mécanique Ondulatoire Appliquée“ in Paris wird unter Leitung von Prof. Dr. R. Lefebvre daran gearbeitet **.

Dessen ungeachtet ist die HFS-Nullfeldresonanz schon jetzt, dank dem vorhandenen Programm von Lefebvre und Maruani, zu einem sehr guten Kontrollverfahren für Hochfeldergebnisse geworden, denn bei sehr vielen Substanzen kann von einer Wechselwirkung nur mit Kernspins 1/2 ausgegangen werden. Der Grad der Übereinstimmung zwischen gemessenem Nullfeldspektrum und dem aus Hochfeldwerten errechnetem ist ein $\mathrm{Ma} ß$ für die Genauigkeit der Hochfeldergebnisse. Darüber hinaus bietet die HFS-Nullfeldresonanz eine Hilfe bei der Deutung komplizierter Hochfeldspektren, wie sie z. B. bei bestrahlten organischen Substanzen durch Entstehung mehrerer Radikale vorkommen, denn bei Nullfeldresonanz treten nur solche Radikale in Erscheinung, die eine HFS-Aufspaltung zeigen. Wei-

\footnotetext{
* Die ersten Ergebnisse für den einfachen Fall des Glycylglycins haben die in dieser Arbeit ermittelten Werte bestätigt.
} 
terhin sind die Hochfeldspektren und Nullfeldspektren grundsätzlich voneinander verschieden, so daß Linien, die im ersteren aufeinander fallen, im Nullfeldspektrum unter Umständen getrennt aufgezeichnet werden können.
Herrn Prof. Dr. R. Lefebvre danken wir für die Überlassung eines Rechenprogramms, dem Deutschen Rechenzentrum in Darmstadt für die Durchführung der Rechnungen an der IBM 7094 und der Deutschen Forschungsgemeinschaft für die finanzielle Unterstützung dieser Arbeit,

\title{
The Electrical Conductivity of (Tl-Rb) $\mathrm{NO}_{3}$ and $(\mathrm{Na}-\mathrm{Rb}) \mathrm{NO}_{3}$
}

\author{
Vittoriano Wagner and SANDro Forcheri \\ Electrochemistry Group EURATOM C. C. R., Petten (N.-H.), Nederland \\ (Z. Naturforsch. 23 a, 926-932 [1968] ; received 2 February 1968)
}

The electrical conductivity of molten $(\mathrm{Na}-\mathrm{Rb}) \mathrm{NO}_{3}$ and $(\mathrm{Tl}-\mathrm{Rb}) \mathrm{NO}_{3}$ was determined.

While the equivalent conductivity of the first system shows the usual negative deviation from additivity, that of the second one varies nearly linearly with composition.

After discussing the conductivity isotherms in terms of some proposed models, an excess conductivity isotherm is presented, derived on the basis of simple assumptions about the trend of both cationic mobilities.

In a previous paper ${ }^{1}$ dealing with the conductivity of $(\mathrm{Na}-\mathrm{Tl}) \mathrm{NO}_{3}$, it was shown that the equivalent conductivity isotherms of this system could be qualitatively discussed by taking polarization into account. In a subsequent paper ${ }^{2}$ the internal mo. bility variations of the smaller cation $\left(\mathrm{Na}^{+}\right)$were correlated with the occurence of the polarized $\mathrm{Na}^{+}-\mathrm{NO}_{3}{ }^{-}-\mathrm{Tl}^{+}$triplet.

Polarization occurs because the common anion is subjected in the point-charge approximation, to a net force

$$
F \propto 1 / \lambda_{1}^{2}-1 / \lambda_{2}^{2}
$$

where $\lambda_{1}=r_{1}+r_{\text {an }}$ and $\lambda_{2}=r_{2}+r_{\text {an }}$, by the two opposite cations, the polarization energy being:

$$
E_{(\mathrm{p})}=\frac{1}{2} \cdot \alpha_{\mathrm{an}} \cdot F^{2}
$$

where $\alpha_{\text {an }}=$ anion polarizability ${ }^{3-6}$.

In this work we have studied the conductivities of $(\mathrm{Tl}-\mathrm{Rb}) \mathrm{NO}_{3}$ and $(\mathrm{Na}-\mathrm{Rb}) \mathrm{NO}_{3}$. Because of the similarity of the $\mathrm{Rb}^{+}$and $\mathrm{Tl}^{+}$ionic radii ${ }^{7}$ the net force $F$ is bound to be zero for the system

1 V.W

2 S. Forcheri and V.W W

3 J. Lumsden, Discussion Faraday Soc. 32, 138 [1961].

4 J. Lumsden, Thermodynamics of Molten Salt Mixtures, Academic Press, London 1966, p. 112-114.

5 S. Hafner and N. H. Nachtrieb, J. Chem. Phys. 42, 631 [1965].

${ }^{6}$ C. T. Moynihan and R. W. Laity, J. Phys. Chem. 68, 3312 [1964].
$(\mathrm{Tl}-\mathrm{Rb}) \mathrm{NO}_{3}$. For the same reason the net force $F$ for the system $(\mathrm{Na}-\mathrm{Rb}) \mathrm{NO}_{3}$ is expected to be comparable to that of the $(\mathrm{Na}-\mathrm{Tl}) \mathrm{NO}_{3}$ system ${ }^{1,2}$.

The supposition that the same electrical force is exerted on the anion by the cations $\mathrm{Rb}^{+}$and $\mathrm{Tl}^{+}$, of similar size, but belonging to two different chemical groups, is supported by the circumstance that $\mathrm{Tl}^{+}$compounds display many similarities to those of the alkalis ${ }^{8}$. Moreover these two cations have practically equal ionic mobilities in acqueous solution at infinite dilution ${ }^{9}$, thus indicating that comparable electrostatic ion-dipole interactions with the water molecules occur. In addition the lattice energies of pure solid $\mathrm{RbNO}_{3}$ and $\mathrm{TlNO}_{3}$ differ only by about $5 \%{ }^{10,11}$.

\section{Experimental}

Apparatus and Material. The experimental set up is similar to the one previously employed ${ }^{1}$, the sole difference being the substitution of the fused salt thermostatic bath with an $\mathrm{Al}$ block, divisible into two pieces,

7 A. J. Dеккеr, Solid State Physics, McMillan Co., London 1963, p. 126.

8 H. Remy, Treatise on Inorganic Chemistry, Elsevier Publ. Co., Amsterdam 1956, p. 381 ff.

9 See ref. ${ }^{8}$, p. 157 and 382.

10 M. F. C. Ladd and W. H. Lee, J. Inorg. Nucl. Chem. 13, 218 [1960].

11 T. C. W Wdington, Advan. Inorganic Chemistry and Radiochemistry, Academic Press, New York 1956, p. 157. 